\title{
Simultaneous Detection of VEGF and CEA by Time-Resolved Chemiluminescence Enzyme-Linked Aptamer Assay
}

This article was published in the following Dove Press journal: International Journal of Nanomedicine

\author{
Jin Man' \\ Jiajia Dong ${ }^{2}$ \\ Yilin Wang ${ }^{\prime}$ \\ Leiliang $\mathrm{He}^{\prime}$ \\ Songcheng $\mathrm{Yu}^{\prime}$ \\ Fei Yu' \\ Jia Wang' \\ Yongmei Tian' \\ Lie Liu' \\ Runping $\mathrm{Han}^{2}$ \\ Hongchao Guo' \\ Yongjun $\mathrm{Wu}\left(\mathbb{I}^{1,3}\right.$ \\ Lingbo $\mathrm{Qu}^{2,4}$
}

\begin{abstract}
'College of Public Health, Zhengzhou University, Zhengzhou, Henan 45000I, People's Republic of China; ${ }^{2}$ College of Chemistry, Zhengzhou University, Zhengzhou, Henan 45000I, People's Republic of China; ${ }^{3}$ The Key Laboratory of Nanomedicine and Health Inspection of Zhengzhou, Zhengzhou, Henan 45000I, People's Republic of China; ${ }^{4}$ Henan Joint International Research Laboratory of Green Construction of Functional Molecules and Their Bioanalytical Applications, Zhengzhou, Henan 45000I, People's Republic of China
\end{abstract}

Correspondence: Yongjun Wu College of Public Health, Zhengzhou University, No. 100 Science Avenue,

Zhengzhou City 45000I, People's

Republic of China

$\mathrm{Tel} / \mathrm{Fax}+86-37|-6778| 450$

Email wuyongjun I35@I26.com

Lingbo Qu

College of Chemistry, Zhengzhou

University, No. 100 Science Avenue,

Zhengzhou City 45000I, People's

Republic of China

$\mathrm{Tel} / \mathrm{Fax}+86-37 \mathrm{I}-6778 I 450$

Email qulingbo@zzu.edu.cn
Background: As two important tumor markers, vascular endothelial growth factor (VEGF) and carcinoembryonic antigen (CEA) have a great value for clinical application in the early diagnosis of cancer. Due to the complex composition of biological samples, the results from combined detection of CEA and VEGF are often taken as a comprehensive indicator in order to make an accurate judgment on a disease. However, most of the current methods can only be used to detect the content of one biomarker. Therefore, it is necessary to explore a simple, rapid, low-cost, and highly sensitive method for the simultaneous detection of CEA and VEGF.

Methods: Based on specific aptamers and magnetic separation, a time-resolved chemiluminescence enzyme-linked aptamer assay was developed for the simultaneous detections of CEA and VEGF in serum samples.

Results: Under the optimal conditions, the linear range of the calibration curve for VEGF was from 0.5 to $80 \mathrm{ng} \mathrm{mL}^{-1}$, and the limit of detection was $0.1 \mathrm{ng} \mathrm{mL}^{-1}$. The linear range of the calibration curve for CEA was 0.5 to $160 \mathrm{ng} \mathrm{mL}^{-1}$, and the limit of detection was 0.1 $\mathrm{ng} \mathrm{mL} \mathrm{m}^{-1}$. The established method was applied to detect VEGF and CEA in serum samples. The results were consistent with those of commercial kits.

Conclusion: The method has high sensitivity and can quickly obtain accurate results, which could greatly improve the measurement efficiency, reduce the cost, and also reduce the volume of sample consumed. It can be seen that the method established in this study has important application value and broad application prospect in clinical diagnosis.

Keywords: chemiluminescence assay, simultaneous detection, vascular endothelial growth factor, time-resolved, carcinoembryonic antigen

\section{Introduction}

Vascular endothelial growth factor (VEGF), which is involved in the regeneration of benign blood vessels and tumor blood vessels, ${ }^{1-3}$ is a key regulator of embryogenesis and vascular physiological development. ${ }^{4}$ In mammals, the VEGF family generally consists of five members, ie, placenta growth factor (PIGF), VEGF-A, VEGF-B, VEGF$\mathrm{C}$, and VEGF-D. ${ }^{5-7}$ There are four different subtypes of VEGF-A, which includes $\mathrm{VEGF}_{121}, \mathrm{VEGF}_{165}, \mathrm{VEGF}_{189}$, and $\mathrm{VEGF}_{206}{ }^{8}$ As the main subtype of the VEGF family, VEGF $_{165}$ may be abnormally expressed in individuals with cancers or diseases. An example is its high expression in the blood or serum of patients with lung cancer and the low expression in patients with psoriasis and Parkinson's disease. ${ }^{9}$ Carcinoembryonic antigen (CEA) is one of the most widely used tumor markers in 
clinical diagnosis, ${ }^{10}$ whose main function is to perform the adhesion reaction between cancer cells and stromal collagen, and also plays an important role in the growth and metastasis of tumors. The level of CEA in serum is low in healthy population, but an elevated CEA level can be found in a variety of cancers like that of the breast, colon, and lung. ${ }^{11,12}$ The development of detection techniques for CEA and VEGF, which are two critical tumor markers, will provide a great application value in the early diagnosis of cancer. ${ }^{13,14}$

Several methods such as fluorescence immunoassay, ${ }^{15,16}$ surface plasmon resonance, ${ }^{17}$ chemiluminescence immunoassay, ${ }^{18,19}$ electrochemical analysis, ${ }^{20}$ etc., have been established for the detection of VEGF and CEA. However, most of the current methods can only detect the content of one component. ${ }^{21-23}$ Due to the complex composition of biological samples, it is difficult to make an accurate judgment of a disease just by detecting the expression level of one marker. As a result, in clinical diagnosis, singletarget detection often results in high probability of misdiagnosis. $^{24}$ Therefore, the development of a simultaneous detection method for the detection of multiple targets has become very important. ${ }^{25}$ The results from the combined detection of CEA and VEGF are often taken as a comprehensive indicator of a certain type of cancer. ${ }^{26,27}$ In practical application, in order to achieve the simultaneous detection of VEGF and CEA, a variety of commercial kits must be purchased to analyze these two components, respectively, making the analysis process cumbersome, costly, and also requires large sample volume. Consequently, it is in urgent need to explore a simple, rapid, low-cost, and highly sensitive method to simultaneously detect CEA and VEGF, which will not only improve the accuracy and reliability of early diagnosis of lung cancer but also play an extremely important role in improving the survival rate of patients.

Chemiluminescent enzyme-linked immunoassay (CLEIA) has been widely used in analytical chemistry and food safety owing to its wide dynamic range, low background interference, high sensitivity, and specificity. ${ }^{28-30}$ The traditional CLEIA is used to mark the catalyst or luminescent agent on the antigen or antibody. After a series of immune reactions, the content of the target is finally detected by measuring chemiluminescence (CL) signal. ${ }^{31,32}$ This method usually requires a large number of antibodies, which are costly and difficult to preserve. In recent years, the nucleic acid aptamer has become an excellent substitute for antibody because of its advantages of low synthesis cost, stable structure, low immunogenicity, and low toxicity. ${ }^{33-35}$ As a consequence, after carefully considering its unique properties and advantages, aptamer rather than antibody was selected to specifically recognize the target in this work. Using systematic evolution of ligands by exponential enrichment (SELEX) strategies, a variety of $\mathrm{VEGF}_{165}$ and CEA aptamers have been identified by previous studies. ${ }^{36-38}$ Referring to the existing studies of $\mathrm{VEGF}_{165}$ and CEA aptamers, the aptamer sequences designed in this study are shown in Table $1 .^{39-42}$ The $\mathrm{VEGF}_{165}$ and CEA aptamers have been proved to bind to their corresponding targets with high affinity, respectively. And the great dissociation constant $(\mathrm{Kd})$ values of the $\mathrm{VEGF}_{165}$ and CEA aptamers were indeed already demonstrated, with a $\mathrm{Kd}$ value of $37.9 \mathrm{nM}$ for $\mathrm{VEGF}_{165}$ and $5 \mathrm{nM}$ for CEA. ${ }^{39,43,44}$ At the same time, on the basis of the aptamers reported in the previous literatures, we added 10 T-base linkers at the 5', which can effectively reduce steric hindrance and facilitate the binding of aptamers to the target.

Based on specific aptamers and magnetic separation, we successfully developed a time-resolved chemiluminescence enzyme-linked immunoassay for the simultaneous quantitative detection of CEA and $\mathrm{VEGF}_{165}$ in real human serum samples. The method established in this study was further used for the quantitative analysis of $\mathrm{VEGF}_{165}$ and CEA in serum samples and compared with the extensively used commercial enzyme-linked immunosorbent assay (ELISA) kits. The results showed that there was no significant difference between CLEIA and commercial ELISA kits. The proposed method can quickly obtain accurate results and achieve high sensitivity, a wider detection range, and lower detection limits. It can be seen that the method established in this

Table I Oligonucleotides Used in This Experiment

\begin{tabular}{|l|l|}
\hline Oligonucleotide & Sequence $\left(\mathbf{5}^{\prime}\right.$ 'to $\mathbf{3}^{\prime}$ ) \\
\hline Complementary DNA probel (cDNAI) & Biotin-TTTTTTTTTTTACCCACCATA \\
Complementary DNA probe2 (cDNA2) & Biotin-TTTTTTTTTTAATTGAATAA \\
VEGFI65 aptamer I (Apt I-HRP) & HRP-TTTTTTTTTTCAATTGGGCCCGTCCGTATGGTGGGT \\
CEA aptamer2 (Apt2) & Biotin-TTTTTTTTTTATACCAGCTTATTCAATT \\
\hline
\end{tabular}


study has important application value and broad application prospect in clinical diagnosis.

\section{Materials and Methods}

\section{Reagents and Materials}

Human VEGF $_{165}$ was purchased from Peprotech Corporation (USA). CEA was obtained from Abcam Corporation (USA). Human CEA ELISA kit was acquired from Huaketai Biotechnology Co. Ltd. (Beijing, China). Human VEGF ${ }_{165}$ ELISA kit was from Miblo Co. Ltd. (Shanghai, China). Alkaline phosphataselabeled streptavidin (ALP-SA) was purchased from Beyotime Biotechnology Co. Ltd. (Shanghai, China). Tween-20, Biotin, Dimethyl sulfoxide (DMSO) and Tris (hydroxymethyl) aminomethane-hydrochloric acid (Tris-HCl) were obtained from Solarbio Co. Ltd. (Beijing, China). $\mathrm{HCl}, \mathrm{NaCl}, \mathrm{KCl}$, and $\mathrm{NaOH}$ were acquired from Kemiou Chemical Reagent Co. Ltd. (Tianjin, China). Streptavidin modified magnetic beads (MBs, $300 \mathrm{~nm}$ ) were from Biomag Biotechnology Co. Ltd. (Wuxi, China). Luminol was acquired from Sigma corporation (USA). 3-(2-Spiroadamatane)-4-methoxy4-(3-phosphoryloxy)phenyl-1,2-dioxetane

(AMPPD) was from Huaxia reagent Co. Ltd. (Chengdu, China). 4-Phenylphenol (BIP) was purchased from Aladdin Co.

Ltd. (Shanghai, China). Ultrapure water (18.3 MU cm) was obtained from a Milli-Q system (Waters, Milford, MA, USA) and used for all experiments.

All oligonucleotides employed in the work were synthesized and purified by Sangon Biotechnology Corporation (Shanghai, China) and the sequences are listed in Table 1.

\section{Instruments}

Centro LB960 chemiluminescence measuring instrument (Berthold, German) was used for chemiluminescence signal recording in this assay. The morphological images of the detection probes were taken by a scanning electron microscope (Hitachi, Japan). Dynamic light scattering (DLS) measurements were realized by Zetasizer Nano-zs 90 particle size and zeta potential analyzer (Malvern, England).

\section{Solution Preparation}

Chemiluminescence substrate A: $1.4 \mathrm{~mL}$ of luminol and $28.6 \mu \mathrm{L}$ of 4-Phenylphenol (BIP) were measured. Then, $0.1 \mathrm{~mol} \mathrm{~L}^{-1}$ Tris-HCl buffer ( $\mathrm{pH}: 8.5$ ) was used to achieve a final volume of $10 \mathrm{~mL}$.
Chemiluminescence substrate B: $25.5 \mu \mathrm{L}$ of $\mathrm{H}_{2} \mathrm{O}_{2}(30 \%)$ was diluted with Milli-Q water to a volume of $25 \mathrm{~mL}$.

Chemiluminescence substrate C: $200 \mu \mathrm{L}$ of AMPPD was measured and diluted with $0.1 \mathrm{~mol} \mathrm{~L}{ }^{-1}$ Tris- $\mathrm{HCl}$ buffer (pH: 8.5) to a concentration of $1 \mathrm{mg} \mathrm{mL}^{-1}$.

Chemiluminescence substrate D: Chemiluminescence substrate $\mathrm{B}$ and chemiluminescence substrate $\mathrm{C}$ were mixed in a volume ratio of $1: 1$.

\section{Principle and Procedure of CLEIA}

The principle of CLEIA is shown in Scheme 1.First, the complementary DNA (cDNA1) that has been biotinylated, was modified on streptavidin-modified magnetic beads (MBs) to synthesize the cDNA1-MBs complex. After the addition of the horseradish peroxidase (HRP)Apt1 (Apt1-HRP) complex and also the $\mathrm{VEGF}_{165}$ detection probe, cDNA1-MBs@Apt1-HRP was formed from the hybridization of the cDNA1-MBs complex with the Apt1-HRP complex. Then, another biotinylated complementary DNA (cDNA2) was modified on the MBs using the same method as described above. After, the addition of the alkaline phosphatase (ALP)-Apt2 (Apt2-ALP) complex and the CEA detection probe led to the synthesis of cDNA2-MBs@Apt2-ALP under the action of base complementation. Then, the two detection probes were mixed in the volume ratio of 1:1 to form the mixed probe. In the presence of $\mathrm{VEGF}_{165}$ and CEA, the two probes specifically bound to the target compounds, to free ALP and HRP labeled on the magnetic beads. Finally, by adding the chemiluminescence substrate, the chemiluminescence system of the Luminol and $\mathrm{H}_{2} \mathrm{O}_{2}$ was catalyzed by the remaining $\mathrm{HRP}$ on the MBs, while the chemiluminescence system of 3-(2-Spiroadamatane)-4-methoxy-4-(3-phosphoryloxy) phenyl-1,2-dioxetane (AMPPD) was catalyzed by the remaining ALP on the MBs. To achieve the simultaneous detections of CEA and VEGF, chemiluminescence signals were, respectively, recorded at different times according to the kinetic characteristics of the different chemiluminescence reactions catalyzed by HRP and ALP.

\section{Preparation of cDNAI-MBs@AptI-HRP} cDNA1 solution $\left(400 \mathrm{nmol} \mathrm{L}{ }^{-1}\right)$ was heated at $95^{\circ} \mathrm{C}$ for 3 min and then quickly placed at $4{ }^{\circ} \mathrm{C}$ to cool down to room temperature. After that, $300 \mu \mathrm{L}$ streptavidincoated magnetic beads were added to a centrifuge tube and washed twice by Tris- $\mathrm{HCl}$ buffer solution with 


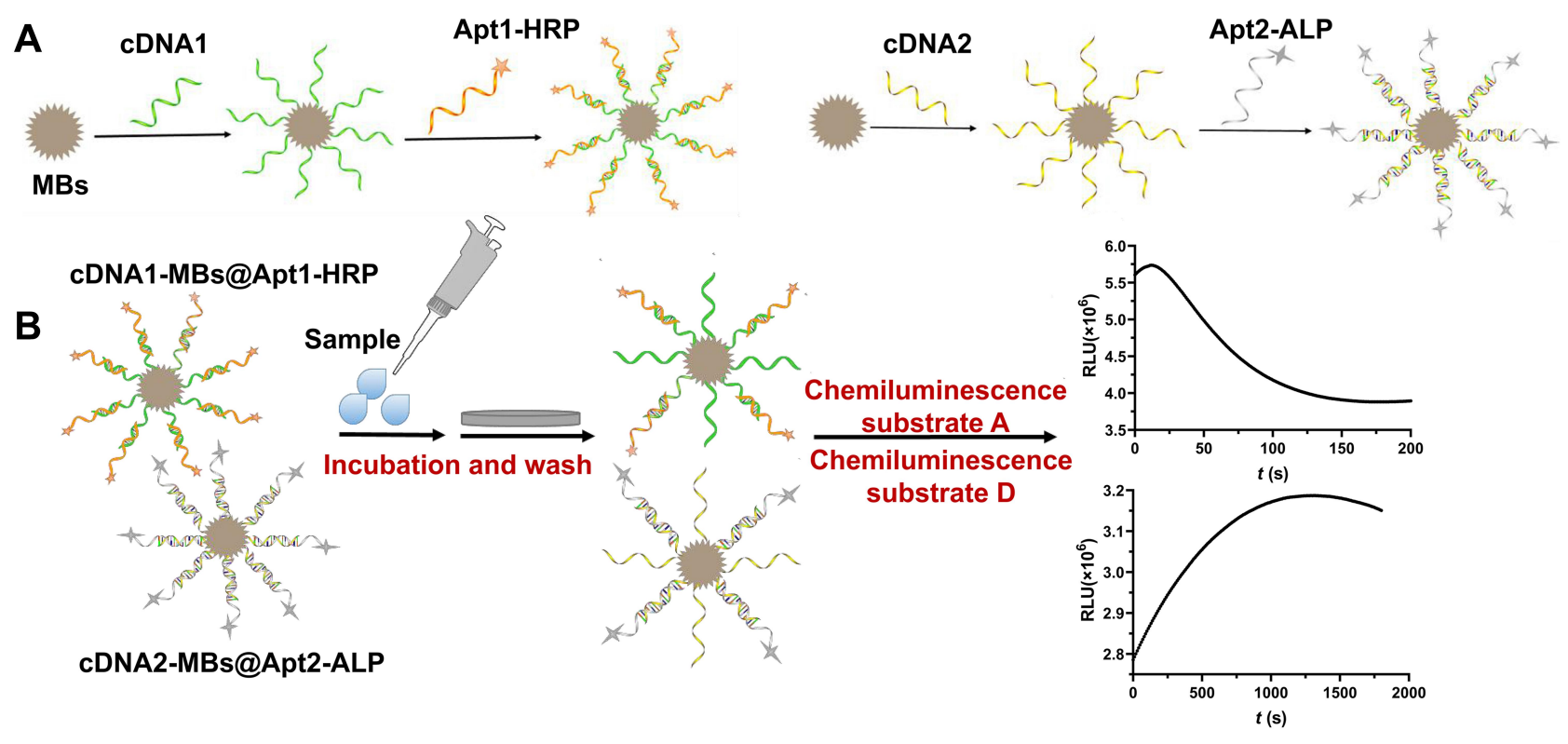

Scheme I Schematic illustration of the simultaneous detection of VEGFI65 and CEA by time-resolved chemiluminescence enzyme aptamer method. (A) cDNAI and cDNA2 immobilization on MBs and coupling with Apt I-HRP and Apt2-ALP to obtain detection probes respectively. (B) Samples were incubated with the mixed probe solution and Chemiluminescence signals were respectively recorded at different times.

Tween-20 (TBST). Then, excess supernatant was removed by magnetic separation. Next, $300 \mu \mathrm{L}$ of the prepared cDNA1 suspension (400 nmol $\mathrm{L}^{-1}$ ) was vortexed with the $300 \mu \mathrm{L}$ streptavidin-coated magnetic beads at room temperature for $30 \mathrm{~min}$ and the mixed solution was washed thrice with TBST. A magnetic separation was performed to remove the supernatant. Then, Apt1-HRP (60 nmol L $\left.{ }^{-1}\right)$ was added to the centrifuge tube for another $50 \mathrm{~min}$ vortexing and the VEGF $_{165}$ detection probe was obtained after magnetic separation of the supernatant. Finally, cDNA1-MBs (a)Apt1-HRP was kept in $600 \mu \mathrm{L}$ of Tris-HCl buffer solution (TBS) at $4^{\circ} \mathrm{C}$ for subsequent experiments.

\section{Preparation of cDNA2-MBs@Apt2-ALP}

The cDNA2 solution (400 nmol L ${ }^{-1}$ ) was heated at $95^{\circ} \mathrm{C}$ for $3 \mathrm{~min}$, and then placed at $4^{\circ} \mathrm{C}$ to slowly cool to room temperature. Apt2 (100 nmol L $\left.{ }^{-1}\right)$ and ALP-SA were vortexed at room temperature for $20 \mathrm{~min}$ to form Apt2ALP complex. Three hundred microliters streptavidincoated magnetic beads were measured into a centrifuge tube and washed twice with TBST. The supernatant was removed by magnetic separation. Next, $300 \mu \mathrm{L}$ of the prepared cDNA2 suspension (400 nmol $\mathrm{L}^{-1}$ ) was vortexed with $300 \mu \mathrm{L}$ of magnetic beads at room temperature for $30 \mathrm{~min}$. A magnetic separation was performed to remove the supernatant and then washed twice with TBST. Then, to obtain the CEA detection probe, the cDNA2-MBs was washed thrice with TBST and vortexed with Apt2-ALP for another $50 \mathrm{~min}$. Finally, after the supernatant had been removed by magnetic separation, cDNA2-MBs@Apt2-ALP was stored in the same way as mentioned above.

\section{Simultaneous Detection of VEGF $_{165}$ and CEA}

Thirty microliters of the mixed probe solution, which was obtained by mixing the prepared CEA detection probe and VEGF $_{165}$ detection probe in a volume of $1: 1$, was added to each well of a 96-well polystyrene microtiter plate, and then the supernatant was removed by magnetic separation. The standard solution $(100 \mu \mathrm{L})$ of $\mathrm{VEGF}_{165}$ and CEA prepared with different concentrations was measured into each well and incubated at $25^{\circ} \mathrm{C}$ for $30 \mathrm{~min}$. After that, the mixed probe was washed thrice with TBST and the supernatant was removed by magnetic separation. Lastly, the chemiluminescence substrate A $(100 \mu \mathrm{L})$ and chemiluminescence substrate D $(100 \mu \mathrm{L})$ were added into the microplate, and the CL signal in each well was recorded at 1.0 $\mathrm{s}$ and $20 \mathrm{~min}$, respectively, using the CL immunoassay analyzer. 


\section{Collection and Detection of Serum Samples}

Fasting peripheral blood was collected with a serum separator tube. The blood samples were bathed at $37^{\circ} \mathrm{C}$ for $30 \mathrm{~min}$ and centrifuged at $3000 \mathrm{rpm}$ for $5 \mathrm{~min}$. Then, serum was collected and CEA and $\mathrm{VEGF}_{165}$ in the serum samples were detected according to the established method developed in this work and by commercial ELISA kits. All blood samples were obtained from the First Affiliated Hospital of Zhengzhou University, approved by LifeScience Ethics Review Committee of Zhengzhou University. All blood donors provided informed consent, in accordance with the Declaration of Helsinki.

\section{Results}

\section{Characteristics of cDNAI-MBs@Apt l- HRP}

Zeta potential and DLS were performed to confirm the conjugation of cDNA1and MBs. As shown in Figure $\underline{\mathrm{S} 1 \mathrm{~A}}$ and Figure S1B , the hydrodynamic diameter of MBs was estimated to be $345.3 \mathrm{~nm}$ after modification with cDNA1, but only $320.3 \mathrm{~nm}$ before modification.
The zeta potential changed from $-4.16 \mathrm{mV}$ to $-16.3 \mathrm{mV}$, indicating the successful formation of the negative cDNA1 polymerization shell on the surface of MBs, and this led to an increase in electrostatic repulsion between particles and a significant improvement in the stability of MBs (Figure

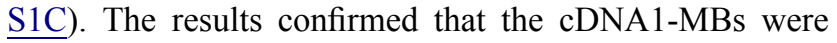
successfully prepared. In addition, the morphology of the prepared cDNA1-MBs@Apt1-HRP complex was characterized by scanning electron microscopy (SEM). The result showed that MBs were spherical, with a flat surface and their diameter were about $300 \mathrm{~nm}$ (Figure 1A). Due to the modification of Apt1-HRP on the MBs, the margin of the cDNA1-MBs@Apt1-HRP complex was not clear (Figure 1B). This primarily indicated that the cDNA1-MBs @.Apt1-HRP was successfully prepared.

The catalytic activity of HRP in cDNA1-MBs@Apt1HRP was investigated by mixing different contents of cDNA1-MBs@Apt1-HRP with chemiluminescent substrate (Figure 2A). The chemiluminescence intensity was enhanced with an increase in the dosage of cDNA1-MBs @ Apt1-HRP, which indicated that HRP maintained a good catalytic activity in the complex, and that cDNA1-MBs @ Apt1-HRP could be used for the detection of $\mathrm{VEGF}_{165}$.
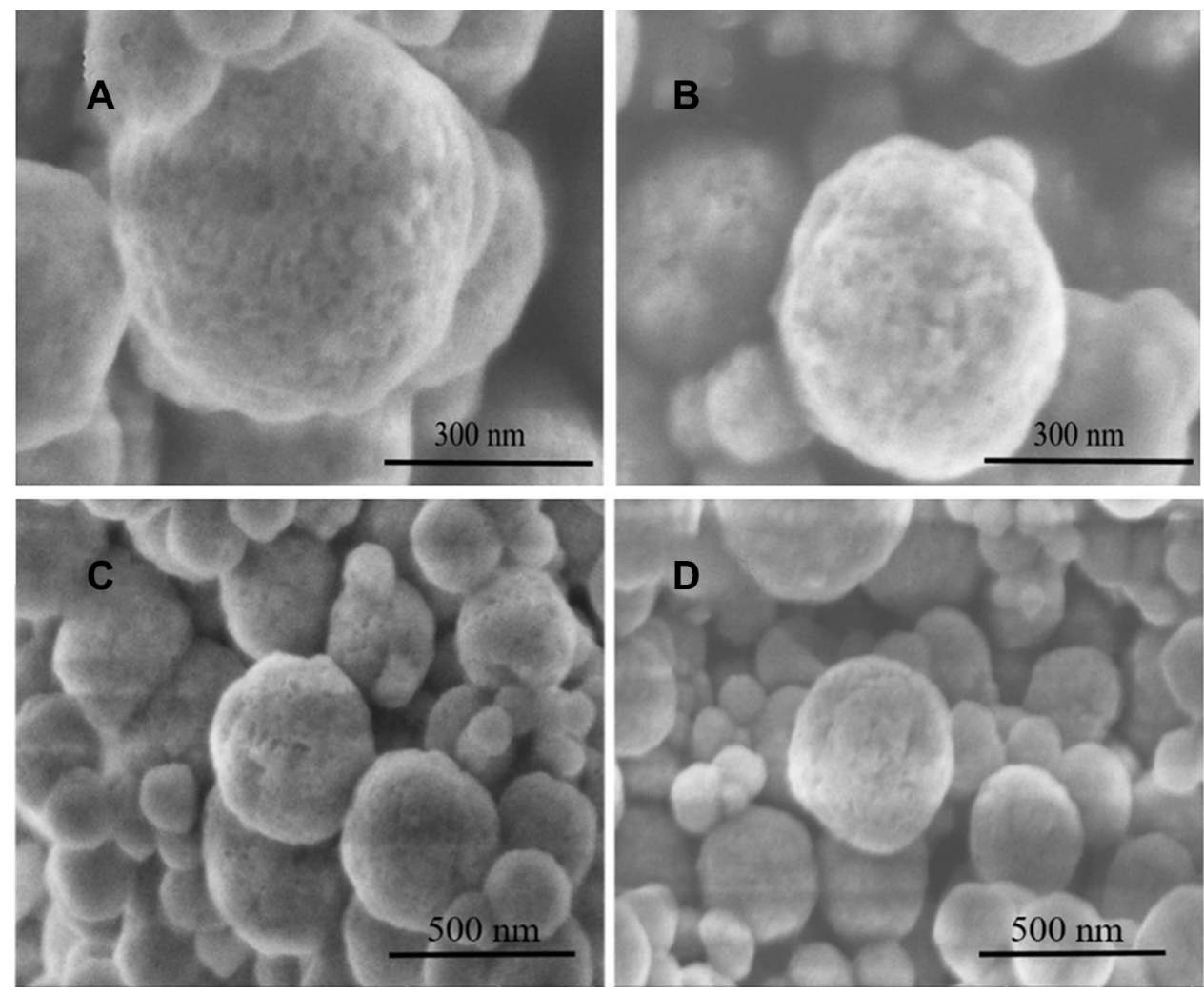

Figure I Scanning electron micrographs of (A) MBs, (B) cDNAI-MBs@AptI-HRP, (Scale bar was 300 nm) (C) MBs and (D) cDNA2-MBs@Apt2-ALP (Scale bar was 500 $\mathrm{nm})$. 

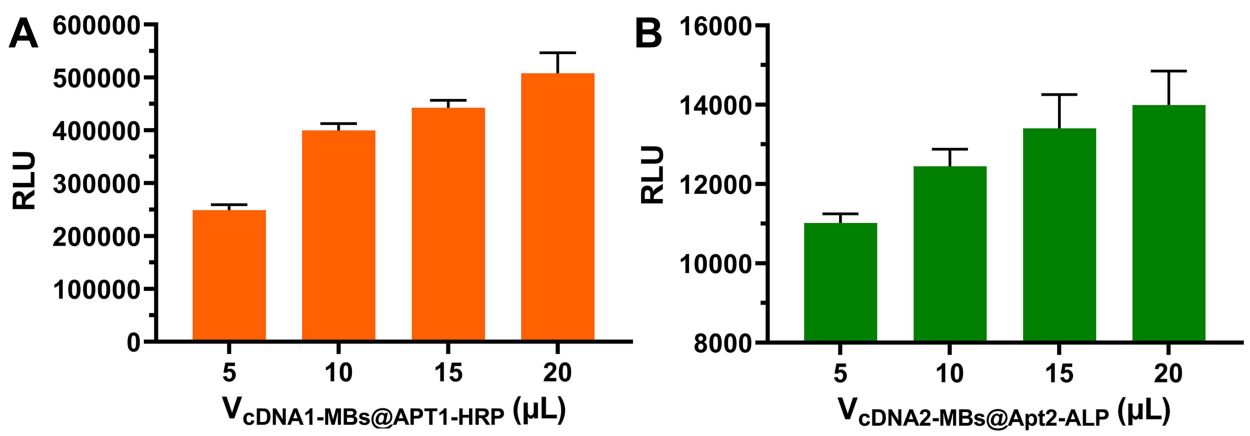

Figure 2 Enzymatic catalytic activity of (A) cDNAI-MBs@AptI-HRP and (B) cDNA2-MBs@Apt2-ALP.

\section{Characteristics of cDNA2-MBs@Apt2-} ALP

After the MBs were modified with cDNA2, the hydrodynamic diameter of the formed cDNA2-MBs showed a significant enhancement from $331.2 \mathrm{~nm}$ to $357.9 \mathrm{~nm}$ (Figures S2A and Figure S2B) and the zeta potential changed from $-4.16 \mathrm{mV}$ to $-12 \mathrm{mV}$ (Figure $\mathrm{S} 2 \mathrm{C}$ ). Therefore, both results confirmed that the cDNA2 and MBs were successfully combined. The results from the scanning electron microscopy of MBs and cDNA2-MBs (a)Apt2-ALP are shown in Figure 1C and D, respectively. Both MBs and cDNA2-MBs@Apt2-ALP were spherical with their diameters around $300 \mathrm{~nm}$. Compared with MBs, the surface of cDNA2-MBs @Apt2-ALP was covered with a layer of material, inferring that the modified Apt2-ALP may be on the surface of the magnetic beads, which indicated that the nanoparticles of cDNA2-MBs@Apt2-ALP were successfully prepared.

The catalytic activity of ALP in cDNA2-MBs@Apt2ALP was investigated by mixing different amounts of cDNA2-MBs@Apt2-ALP with chemiluminescence substrate and the changes in chemiluminescence intensity were observed (Figure 2B). As the amount of cDNA2MBs@Apt2-ALP increased, the chemiluminescence intensity and the relative chemiluminescence units (RLU) continued to rise, suggesting that the prepared CEA detection probe maintained a good catalytic activity of ALP and could be applied in subsequent experiments.

\section{Experimental Conditions Optimization}

In order to achieve a better sensing effect, the following parameters were optimized in this assay: Concentration of the Apt1-HRP, dilution ratio of the ALP-SA, concentration of the Apt2, reaction time between aptamers and complementary DNA, and reaction time between detection probes and samples, concentration of the luminol, $\mathrm{H}_{2} \mathrm{O}_{2}$, and AMPPD.

As displayed in Figure 3A, with an increase in the concentration of Apt1-HRP, $R U_{0}-R L U$ increased at the beginning and reached a maximum at $60 \mathrm{nmol} \mathrm{L}^{-1}$. Therefore, $60 \mathrm{nmol} \mathrm{L}^{-1}$ of Apt1-HRP was selected as the optimal concentration. Figure $3 \mathrm{~B}$ indicated that with a decrease in the dilution rate of ALP-SA from 1:100 to $1: 200$, the $R_{L} U_{0}-R L U$ increased slightly, and when the dilution ratio of the ALP-SA was lower than 1:200, the signal observably declined. Therefore, we set the dilution ratio of the ALP-SA as 1:200. The results (shown in Figure 3C) also demonstrated that the optimal concentration of the Apt2 was $100 \mathrm{nmol} \mathrm{L}^{-1}$.

Meanwhile, the reaction times between aptamers and complementary DNA, and also between the detection probes and samples were optimized. As shown in Figure $\underline{\text { S3}}$, signal intensity at the beginning, increased gradually with an increasing reaction time, but after $50 \mathrm{~min}$ and 30 min, respectively, $\mathrm{RLU}_{0}-\mathrm{RLU}$ decreased steadily. Therefore, the optimal reaction times between aptamers and complementary DNA, detection probes and samples were $50 \mathrm{~min}$ and $30 \mathrm{~min}$, respectively.

Because the substrates of the chemiluminescence and the concentration of the luminol, $\mathrm{H}_{2} \mathrm{O}_{2}$, and AMPPD can effectively affect the intensity of chemiluminescence, we investigated the optimal concentration of luminol, $\mathrm{H}_{2} \mathrm{O}_{2}$, and AMPPD. As shown in Figure 3D-F, the RLU of luminol, $\mathrm{H}_{2} \mathrm{O}_{2}$, and AMPPD increased sharply with the concentration and tended to level off after $1.4 \mathrm{mmol} \mathrm{L}^{-1}, 5$ $\mathrm{mmol} \mathrm{L}^{-1}$, and $0.5 \mathrm{mg} \mathrm{mL}^{-1}$, respectively. Therefore, 1.4 $\mathrm{mmol} \mathrm{L}{ }^{-1}, 5 \mathrm{mmol} \mathrm{L}^{-1}$, and $0.5 \mathrm{mg} \mathrm{mL}^{-1}$ were chosen as the optimal concentration of luminol, $\mathrm{H}_{2} \mathrm{O}_{2}$, and AMPPD, respectively. 

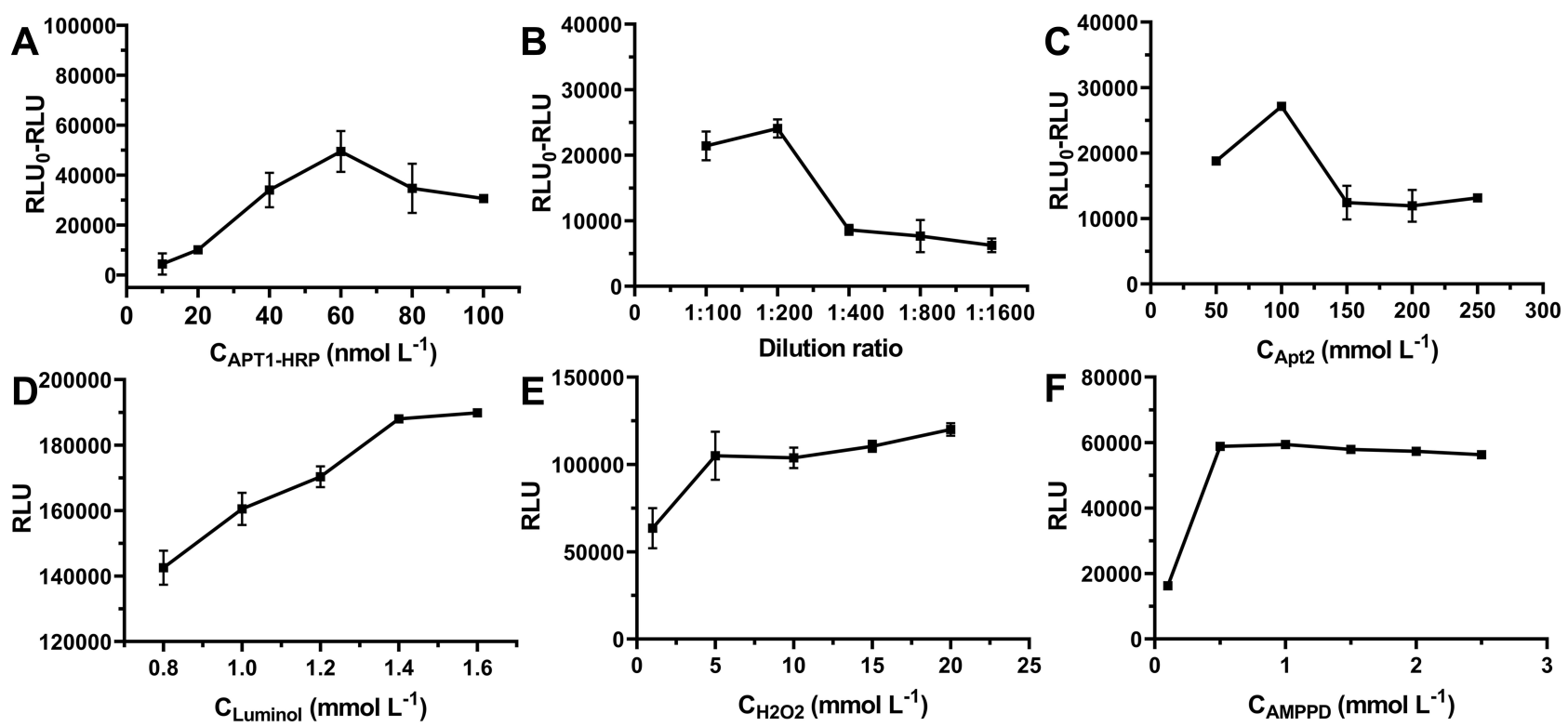

Figure 3 Experimental conditions optimization. (A) Optimization of the concentration of the Apt I-HRP. (B) Optimization of the dilution ratio of the ALP-SA. Optimization of the concentration of the the Apt2 (C), luminol (D), $\mathrm{H}_{2} \mathrm{O}_{2}(\mathbf{E})$, and AMPPD (F).

\section{Kinetic Characteristics of Different}

\section{Chemiluminescent Systems}

The chemiluminescence kinetic characteristics of the two systems, ALP-AMPPD and HRP-Luminol- $\mathrm{H}_{2} \mathrm{O}_{2}$, were investigated (Figure 4). The kinetic characteristics of the HRP-Luminol- $\mathrm{H}_{2} \mathrm{O}_{2}$ system are shown in Figure 4A. The system produced a flash-type chemiluminescence, the intensity of which was high in the first $10 \mathrm{~s}$ of the reaction and decayed rapidly thereafter. Then, we chose $1.0 \mathrm{~s}$ as the time window for detecting HRP-Luminol- $\mathrm{H}_{2} \mathrm{O}_{2}$ system. As shown in Figure 4B, the ALP-AMPPD system produced a glow-type chemiluminescence. The chemiluminescence intensity was low at the beginning of the reaction, then increased slowly, reaching the maximum luminous intensity at about $20 \mathrm{~min}$. It then maintained a plateau for a period, and then decreased slowly thereafter. Therefore, $20 \mathrm{~min}$ was chosen as the time window for detecting the ALP-AMPPD system.

\section{Analytical Performance of the Assay}

A time-resolved CLEIA for the simultaneous quantitative detection of CEA and VEGF $_{165}$ was established under the optimal conditions. Calibration curves were obtained by plotting chemiluminescence RLU values against different concentrations $\left(0.5,1,5,10,20,40,80,160 \mathrm{ng} \mathrm{mL}^{-1}\right)$ of standard $\mathrm{VEGF}_{165}$ and CEA sample. As observed from Figure 5A, the CL intensity was linear with $\operatorname{lgCVEGF}_{165}$ $[Y=0.2603 X+4.3684$ ( $Y$ and $X$, respectively, denote the $\lg \left(\mathrm{RLU}_{0}-\mathrm{RLU}\right)$ and $\left.\operatorname{lgCVEGF}_{165}, \mathrm{r}=0.9882\right]$ in the range of $0.5 \mathrm{ng} \mathrm{mL} \mathrm{m}^{-1}$ and $80 \mathrm{ng} \mathrm{mL}^{-1}$, with the limit of detection
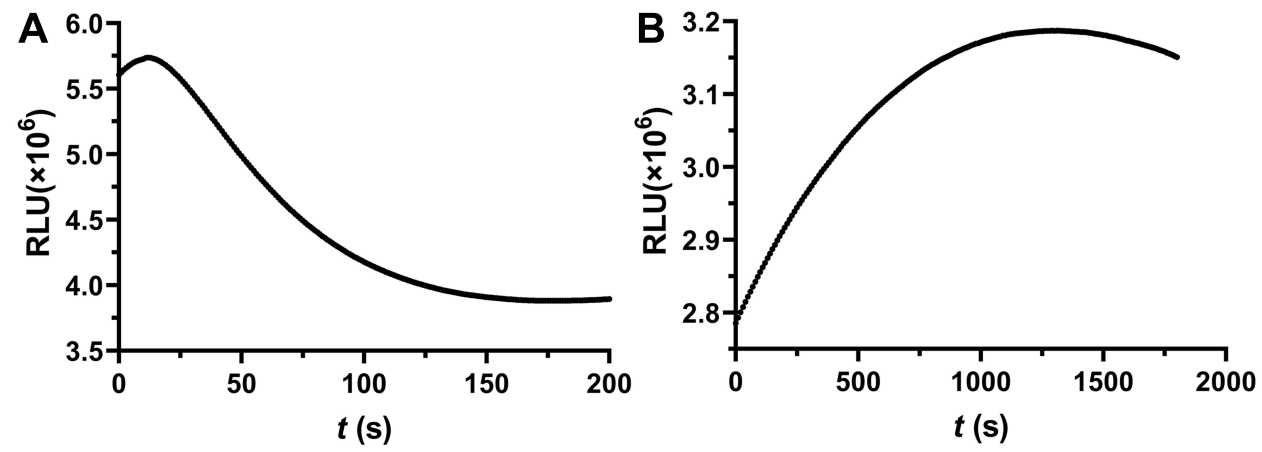

Figure 4 Kinetics curve of (A) HRP-Luminol- $\mathrm{H}_{2} \mathrm{O}_{2}$ and (B) ALP-AMPPD chemiluminescent systems. 

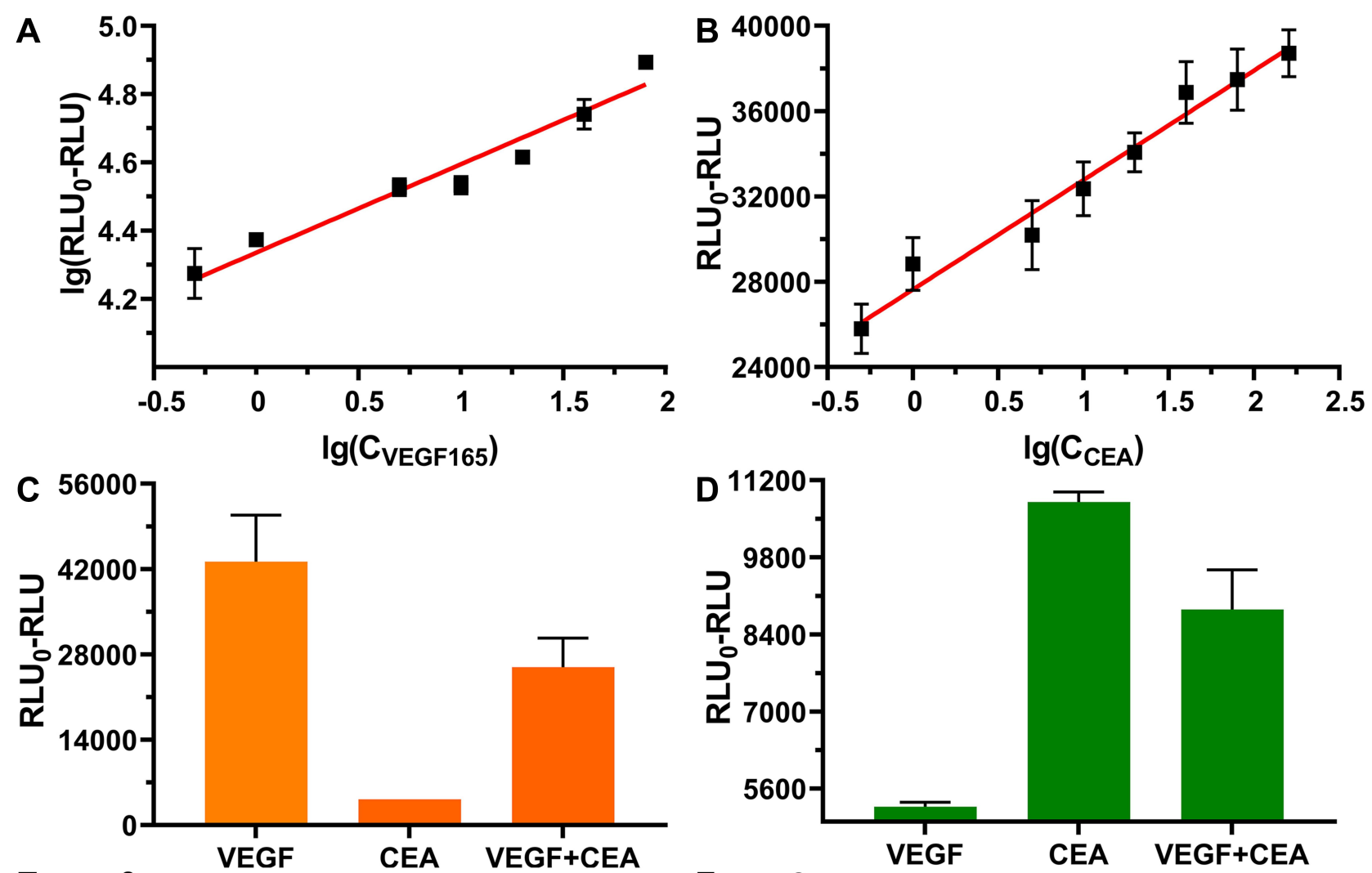

E

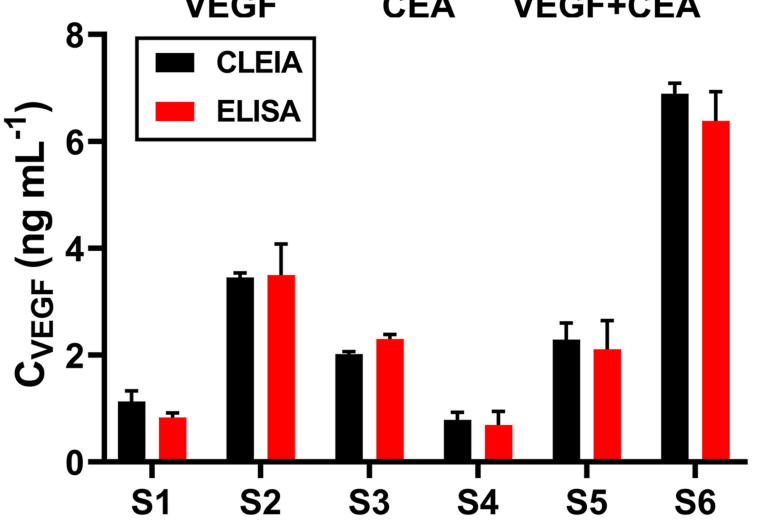

$\mathbf{F}$

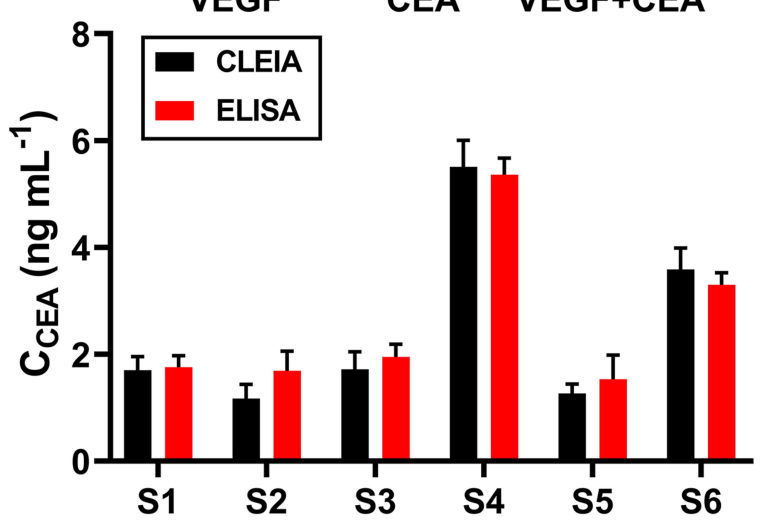

The number of real serum samples

Figure 5 Calibration curves for (A) VEGF ${ }_{165}$ and (B) CEA determination. The concentrations of VEGF 165 and CEA are both $0.5, \mathrm{I}_{1}, 5,10,20,40,80,160 \mathrm{ng}^{-1}$, respectively. Chemiluminescence signal detected at (C) $1.0 \mathrm{~s}$ and (D) $20 \mathrm{~min}$ (The concentrations of VEGF 165 and CEA are both $50 \mathrm{ng} \mathrm{mL}^{-1}$ ). The detection results of (E) VEGF $_{165}$ and CEA (F) in serum samples.

(LOD) of $0.1 \mathrm{ng} \mathrm{mL}^{-1}$. Figure $5 \mathrm{~B}$ also showed that there was a linear intensity when the concentration ranged from $0.5 \mathrm{ng} \mathrm{mL}^{-1}$ to $160 \mathrm{ng} \mathrm{mL}^{-1}$ with a LOD of $0.1 \mathrm{ng} \mathrm{mL}^{-1}$. The linear equation was $\mathrm{Y}=5032.03 \mathrm{X}+27732.14$ ( $\mathrm{Y}$ and $\mathrm{X}$, respectively, denote the (RLU $\left.\mathrm{R}_{0} \mathrm{RLU}\right)$ and $\operatorname{lgCCEA}$, $\mathrm{r}=0.9893$ ). The components of serum samples are complex and often contain both VEGF and CEA. In order to evaluate whether this method can be used in actual sample detection, we verified the specificity of the developed method for $\mathrm{VEGF}_{165}$ and CEA detection by measuring the $\mathrm{CL}$ signal response of three targets, CEA (50 $\left.\mathrm{ng} \mathrm{mL}{ }^{-1}\right), \operatorname{VEGF}_{165}\left(50 \mathrm{ng} \mathrm{\textrm {mL } ^ { - 1 } ) \text { and CEA (50 ng mL }}{ }^{-1}\right.$ )$+\mathrm{VEGF}_{165}\left(50 \mathrm{ng} \mathrm{mL}{ }^{-1}\right)$, at $1.0 \mathrm{~s}$ and $20 \mathrm{~min}$, respectively. It can be seen that the $\mathrm{VEGF}_{165}$ group and the $\mathrm{CEA}+\mathrm{VEGF}_{165}$ group had obvious response at $1.0 \mathrm{~s}$ but the signal value of the CEA group was very low (Figure 5C). Significant signal values were observed in the CEA group and the mixed group at 20 min but the $\mathrm{VEGF}_{165}$ 
Table 2 Results of Serum Samples Tested with the CLEIA and ELISA Kit

\begin{tabular}{|l|l|l|l|l|}
\hline \multirow{2}{*}{ Samples } & \multicolumn{2}{l|}{${\text { C CEA }\left(\mathrm{ng} \mathrm{mL}^{-1}\right)}$} & \multicolumn{2}{l|}{ C VEGF $_{\left.\text {(ng } \mathbf{~ m L}^{-1}\right)}$} \\
\cline { 2 - 5 } & CLEIA & ELISA & CLEIA & ELISA \\
\hline SI & 1.70 & 1.76 & 1.13 & 0.83 \\
S2 & 1.17 & 1.69 & 3.45 & 3.50 \\
S3 & 1.72 & 1.95 & 2.02 & 2.30 \\
S4 & 5.51 & 5.36 & 0.79 & 0.69 \\
S5 & 1.27 & 1.53 & 2.29 & 2.11 \\
S6 & 3.59 & 3.30 & 6.89 & 6.38 \\
\hline
\end{tabular}

group hardly responded to the ALP system (Figure 5D). This means the detection probes had no specific reaction to other target object, indicating that this method had good specificity and could achieve simultaneous detection of VEGF and CEA in samples.

\section{Application in Human Serum Samples}

To demonstrate the application of the proposed CLEIA in biological samples, six human serum samples were employed to detect the concentrations of $\mathrm{VEGF}_{165}$ and CEA under the optimum conditions and the results were compared to that of the extensively used ELISA method. The results are summarized in Figure 5 (E-F) and Table 2. Paired sample $t$ test was used to statistically analyze the test results. Furthermore, the results of CEA $(t=0.872$, $P=0.423)$ and $\operatorname{VEGF}_{165}(t=-1.118, P=0.314)$ in serum samples measured by the new chemiluminescence method were not statistically different from those of commercial ELISA kit, suggesting that the method could be applied to detect VEGF 165 and CEA content in serum.

\section{Conclusion}

In this work, we have developed a novel and sensitive time-resolved chemiluminescence enzyme-linked immunoassay for the simultaneous quantitative detection of CEA and VEGF $_{165}$ in human serum samples, based on the different kinetics of chemiluminescence reactions catalyzed by HRP and ALP. The proposed CLEIA revealed wide linear responses $\left(\mathrm{VEGF}_{165}: 0.5 \mathrm{ng} \mathrm{mL}^{-1}-80 \mathrm{ng} \mathrm{mL}^{-1}\right.$, CEA: $0.5 \mathrm{ng} \mathrm{mL}^{-1}-160 \mathrm{ng} \mathrm{mL}^{-1}$ ) toward $\mathrm{VEGF}_{165}$ and CEA with low LODs $\left(\mathrm{VEGF}_{165}: 0.1 \mathrm{ng} \mathrm{mL}^{-1}, \mathrm{CEA}: 0.1\right.$ $\mathrm{ng} \mathrm{mL}^{-1}$ ). More importantly, the proposed method was successfully used for the detection of $\mathrm{VEGF}_{165}$ and CEA in various actual samples and the experimental results were consistent with that of the ELISA kit, indicating that the method had high accuracy. In addition, the assay has many advantages and innovations. First, magnetic beads were selected as solid phase carrier in this study, which, on one hand, achieved rapid separation of reactants from solution, and, on the other hand, enabled biomolecules to be well fixed. Secondly, aptamers with low cost, stable structure, and easy to modify were used to replace the antibodies usually used in traditional CLEIA so as to specifically recognize the target. Meanwhile, the simultaneous detections of CEA and VEGF in serum samples were realized by using the different kinetic characteristics of glow and flash chemiluminescence reaction systems, which did not only save samples but also improved the analysis efficiency. However, there are still several points that need to be improved in the experiment. The limit of detection can be further reduced and also, the sensitivity of multi-component analysis can be improved by using magnetic beads of different materials, by modifying different groups on the magnetic beads, or by blocking the surface of the magnetic beads with some surfactants, etc. Overall, the proposed approach can be used for high-throughput screening and has potential applications in early diagnosis of cancer.

\section{Acknowledgments}

This work was supported by the National Natural Science Foundation of China (grant numbers 81573203, 81973099).

\section{Disclosure}

The authors report no conflicts of interest in this work.

\section{References}

1. Ferrara N. Vascular endothelial growth factor: basic science and clinical progress. Endocr Rev. 2004;25(4):581-611.

2. Roskoski R. Vascular endothelial growth factor (VEGF) signaling in tumor progression. Crit Rev Oncol Hematol. 2007;62(3):179-213. doi:10.1016/j.critrevonc.2007.01.006

3. Rud'ko AS, Efendieva MK, Budzinskaya MV, Karpilova MA. Influence of vascular endothelial growth factor on angiogenesis and neurogenesis. Vestn Oftalmol. 2017;133(3):75-81.

4. Hoeben A, Landuyt B, Highley MS, Wildiers H, Van Oosterom AT, De Bruijn EA. Vascular endothelial growth factor and angiogenesis. Pharmacol Rev. 2004;56(4):549-580.

5. Simons M, Gordon E, Claesson-Welsh L. Mechanisms and regulation of endothelial VEGF receptor signalling. Nat Rev Mol Cell Biol. 2016;17(10):611-625.

6. Ribatti D. The controversial role of placental growth factor in tumor growth. Cancer Lett. 2011;307(1):1-5.

7. Abbink K, Zusterzeel PLM, Geurts-Moespot A, van der Steen R, Span PN, Sweep F. Prognostic significance of VEGF and components of the plasminogen activator system in endometrial cancer. $J$ Cancer Res Clin Oncol. 2020;146(7):1725-1735.

8. Dehghani S, Nosrati R, Yousefi M, et al. Aptamer-based biosensors and nanosensors for the detection of vascular endothelial growth factor (VEGF): A review. Biosens Bioelectron. 2018;110:23-37. 
9. Detmar M. Evidence for vascular endothelial growth factor (VEGF) as a modifier gene in psoriasis. J Investig Dermatol Symp Proc. 2004;122(1):xiv-Xv.

10. Duffy MJ. Carcinoembryonic antigen as a marker for colorectal cancer: is it clinically useful? Clin Chem. 2001;47(4):624-630. doi:10.1093/clinchem/47.4.624

11. Ghosh I, Bhattacharjee D, Das AK, Chakrabarti G, Dasgupta A, Dey SK. Diagnostic Role of Tumour Markers CEA, CA15-3, CA19-9 and CA125 in Lung Cancer. Indian J Clin Biochem. 2013;28(1):24-29. doi:10.1007/s12291-012-0257-0

12. Gu X, She Z, Ma T, Tian S, Kraatz H-B. Electrochemical detection of carcinoembryonic antigen. Biosens Bioelectron. 2018;102:610-616. doi:10.1016/j.bios.2017.12.014

13. Gulhati P, Yin J, Pederson L, et al. Threshold Change in CEA as a Predictor of Non-Progression to First-Line Systemic Therapy in Metastatic Colorectal Cancer Patients With Elevated CEA. J Natl Cancer Inst. 2020;112(11):1127-1136. doi:10.1093/jnci/djaa020

14. May KML, Vogt A, Bachas LG, Anderson KW. Vascular endothelial growth factor as a biomarker for the early detection of cancer using a whole cell-based biosensor. Anal Bioanal Chem. 2005;382 (4):1010-1016. doi:10.1007/s00216-005-3224-2

15. Li J, Sun K, Chen Z, Shi J, Zhou D, Xie G. A fluorescence biosensor for VEGF detection based on DNA assembly structure switching and isothermal amplification. Biosens Bioelectron. 2017;89(Pt 2):964-969. doi:10.1016/j.bios.2016.09.078

16. Danesh NM, Yazdian-Robati R, Ramezani M, Alibolandi M, Abnous K, Taghdisi SM. A label-free aptasensor for carcinoembryonic antigen detection using three-way junction structure and ATMND as a fluorescent probe. Sensors and Actuators B: Chemical. 2018;256:408-412. doi:10.1016/j.snb.2017.10.126

17. Chen H, Hou Y, Qi F, et al. Detection of vascular endothelial growth factor based on rolling circle amplification as a means of signal enhancement in surface plasmon resonance. Biosens Bioelectron. 2014;61:83-87. doi:10.1016/j.bios.2014.05.005

18. Chong J, Chong H, Lee JH. A chemiluminescent dual-aptasensor capable of simultaneously quantifying prostate specific antigen and vascular endothelial growth factor. Anal Biochem. 2019;564:102-107. doi:10.1016/j.ab.2018.10.024

19. Jiang J, Zhao S, Huang Y, Qin G, Ye F. Highly sensitive immunoassay of carcinoembryonic antigen by capillary electrophoresis with gold nanoparticles amplified chemiluminescence detection. J Chromatogr A. 2013;1282:161-166. doi:10.1016/j. chroma.2013.01.066

20. Da H, Liu H, Zheng Y, Yuan R, Chai Y. A highly sensitive VEGF165 photoelectrochemical biosensor fabricated by assembly of aptamer bridged DNA networks. Biosens Bioelectron. 2018;101:213-218. doi:10.1016/j.bios.2017.10.032

21. Wang YG, Zhao GH, Zhang Y, Du B, Wei Q. Ultrasensitive photoelectrochemical immunosensor based on $\mathrm{Cu}$-doped $\mathrm{TiO} 2$ and carbon nitride for detection of carcinoembryonic antigen. Carbon. 2019;146:276-283. doi:10.1016/j.carbon.2019.02.008

22. Shan S, He Z, Mao S, Jie M, Yi L, Lin J-M. Quantitative determination of VEGF165 in cell culture medium by aptamer sandwich based chemiluminescence assay. Talanta. 2017;171:197-203. doi:10.1016/j. talanta.2017.04.057

23. Wu D, Gao T, Lei L, Yang D, Mao X, Li G. Colorimetric detection of proteins based on target-induced activation of aptazyme. Anal Chim Acta. 2016;942:68-73. doi:10.1016/j.aca.2016.09.010

24. Kim YI, Jeong S, Jung KO, et al. Simultaneous Detection of EGFR and VEGF in Colorectal Cancer using Fluorescence-Raman Endoscopy. Sci Rep. 2017;7(1):1035.

25. Pan LH, Kuo SH, Lin TY, Lin CW, Fang PY, Yang HW. An electrochemical biosensor to simultaneously detect VEGF and PSA for early prostate cancer diagnosis based on graphene oxide/ssDNA/PLLA nanoparticles. Biosens Bioelectron. 2017;89(Pt 1):598-605.
26. Dong J, He L, Yu F, et al. Enhanced chemiluminescence enzyme-linked immunoassay for the determination of DNA methyltransferase 1 in human serum. Luminescence. 2019;34(3):368-374.

27. Dbouk HA, Tawil A, Nasr F, Kandakarjian L, Abou-Merhi R. Significance of CEA and VEGF as Diagnostic Markers of Colorectal Cancer in Lebanese Patients. Open Clin Cancer J. 2007;1:1-5.

28. Vdovenko MM, Gribas AV, Vylegzhanina AV, Sakharov IY. Development of a chemiluminescent enzyme immunoassay for the determination of dexamethasone in milk. Anal Methods. 2012;4 (8):2550-2554.

29. Yang JY, Zhang Y, Lei HT, et al. Development of an Ultra-sensitive Chemiluminescence Enzyme Immunoassay for the Determination of Diethylstilbestrol in Seafood. Anal Lett. 2013;46(14):2189-2202.

30. He ST, He LL, Liu BB, et al. Development of a rapid and sensitivity magnetic chemiluminescence immunoassay for DNA methyltransferase 1 in human serum. Chin Chem Lett. 2019;30(5):1031-1034.

31. Yang XY, Guo YS, Bi S, Zhang SS. Ultrasensitive enhanced chemiluminescence enzyme immunoassay for the determination of alpha-fetoprotein amplified by double-codified gold nanoparticles labels. Biosens Bioelectron. 2009;24(8):2707-2711.

32. Wang G, Wan Y, Lin G, Li Z, Dong Z, Liu T. Development of a novel chemiluminescence immunoassay for the detection of procalcitonin. J Immunol Methods. 2020;484-485:112829.

33. Jin C, Qiu L, Li J, Fu T, Zhang X, Tan W. Cancer biomarker discovery using DNA aptamers. Analyst. 2016;141(2):461-466.

34. Qi Y, Song D, Chen Y. Colorimetric oligonucleotide-based sensor for ultra-low $\mathrm{Hg} 2+$ in contaminated environmental medium: convenience, sensitivity and mechanism. Sci Total Environ. 2020;142579.

35. Qi Y, Ma J, Chen X, Xiu FR, Chen Y, Lu Y. Practical aptamer-based assay of heavy metal mercury ion in contaminated environmental samples: convenience and sensitivity. Anal Bioanal Chem. 2020;412 (2):439-448.

36. Hongxia C, Zaijun L, Ruiyi L, Guangli W, Zhiguo G. Molecular machine and gold/graphene quantum dot hybrid based dual amplification strategy for voltammetric detection of VEGF165. Mikrochim Acta. 2019;186(4):242.

37. Zhang H, Li M, Li C, et al. G-quadruplex DNAzyme-based electrochemiluminescence biosensing strategy for VEGF165 detection: combination of aptamer-target recognition and $\mathrm{T} 7$ exonuclease-assisted cycling signal amplification. Biosens Bioelectron. 2015;74:98-103.

38. Zhao L, Cheng M, Liu G, et al. A fluorescent biosensor based on molybdenum disulfide nanosheets and protein aptamer for sensitive detection of carcinoembryonic antigen. Sensors Actuators B Chem. 2018;273:185-190.

39. Riccardi C, Musumeci D, Platella C, Gaglione R, Arciello A, Montesarchio D. Tuning the Polymorphism of the Anti-VEGF G-rich V7t1 Aptamer by Covalent Dimeric Constructs. Int $J$ Mol Sci. 2020;21(6):1963.

40. Xu L, Zou L, Guo J, Cao Y, Feng C, Simple YB. "Signal-Off" Electrochemical Aptasensor Based on Aptamer-Cu3(PO4)2 Hybrid Nanoflowers/Graphene Oxide for Carcinoembryonic Antigen Detection. ChemElectroChem. 2020;7(7):1660-1665.

41. Xiang W, Lv Q, Shi H, Xie B, Gao L. Aptamer-based biosensor for detecting carcinoembryonic antigen. Talanta. 2020;214:120716.

42. Tang H, Wang H, Yang C, Zhao D, Qian Y, Nanopore-based LY. Strategy for Selective Detection of Single Carcinoembryonic Antigen (CEA) Molecules. Anal Chem. 2020;92(4):3042-3049.

43. Smith CLB, Cassandra L. Assignee. Compositions comprising nucleic acid aptamers. United States patent US 20130101506. 2013.

44. Manochehry S, McConnell EM, Li Y. Unraveling Determinants of Affinity Enhancement in Dimeric Aptamers for a Dimeric Protein. Sci Rep. 2019;9(1):17824. 


\section{Publish your work in this journal}

The International Journal of Nanomedicine is an international, peerreviewed journal focusing on the application of nanotechnology in diagnostics, therapeutics, and drug delivery systems throughout the biomedical field. This journal is indexed on PubMed Central, MedLine, CAS, SciSearch ${ }^{\mathbb{B}}$, Current Contents ${ }^{\mathbb{B}} /$ Clinical Medicine, $^{2}$
Journal Citation Reports/Science Edition, EMBase, Scopus and the Elsevier Bibliographic databases. The manuscript management system is completely online and includes a very quick and fair peer-review system, which is all easy to use. Visit http://www.dovepress.com/ testimonials.php to read real quotes from published authors.

Submit your manuscript here: https://www.dovepress.com/international-journal-of-nanomedicine-journal 